\title{
Oblique-mode breakdown in hypersonic and high-enthalpy boundary layers over a blunt cone
}

\author{
Xianliang Chen, Dongxiao Xu and Song Fu* (D)
}

\section{*Correspondence:}

fs-dem@tsinghua.edu.cn

School of Aerospace Engineering,

Tsinghua University, 100084 Beijing,

China

\begin{abstract}
The nonlinear analyses of the hypersonic and high-enthalpy boundary-layer transition had received little attention compared with the widely-studied linear instabilities. In this work, the oblique-mode breakdown, as one of the most available transition mechanisms, is studied using the nonlinear parabolized stability equations (NPSE) with consideration of the thermal-chemical non-equilibrium effects. The flow over a blunt cone is computed at a free-stream Mach-number of 15. The rope-like structures and the spontaneous radiation of sound waves are observed in the schlieren-like picture. It is also illustrated that the disturbances of the species mass and vibrational temperature near the wall are mainly generated by the product term of the wall-normal velocity disturbance and the mean-flow gradient. In comparison to the CPG flow, the TCNE effects destabilize the second mode and push upstream the $N$ factor envelope. The higher growth rate of the oblique wave leads to stronger growth of the streamwise vortices and harmonic waves.
\end{abstract}

Keywords: Boundary-layer transition, Hypersonics, High-enthalpy flows

\section{Introduction}

The accurate prediction and effective control of the boundary-layer transition are especially helpful in designing thermal protection and engine intake systems for high-speed vehicles. However, this transition process is extremely complicated due to its high nonlinearity and sensitivity to many factors [1]. For hypersonic and high-enthalpy boundary layers, the flow transition is even more complex with the appearance of "high-temperature effects" [2]. New gas models are developed, instead of the calorically perfect gas (CPG) assumption, to describe the thermal-chemical non-equilibrium (TCNE) processes [3]. Nevertheless, the influences remain unresolved of high-temperature effects on the boundary-layer instability and transition.

In flight where the free stream is usually "quiet", the natural transition is the most likely route to turbulence [4]. The linear mechanisms are mainly responsible for the quick amplification of the initial disturbances, which are well-described by the linear stability theory (LST) [5]. The LST results show that multiple unstable modes

(c) The Author(s). 2021 Open Access This article is licensed under a Creative Commons Attribution 4.0 International License, which permits use, sharing, adaptation, distribution and reproduction in any medium or format, as long as you give appropriate credit to the original author(s) and the source, provide a link to the Creative Commons licence, and indicate if changes were made. The images or other third party material in this article are included in the article's Creative Commons licence, unless indicated otherwise in a credit line to the material. If material is not included in the article's Creative Commons licence and your intended use is not permitted by statutory regulation or exceeds the permitted use, you will need to obtain permission directly from the copyright holder. To view a copy of this licence, visit http://creativecommons.org/licenses/by/4.0/. 
exist in hypersonic boundary layers where the second-mode instability has the largest growth rate [6]. The most unstable second-mode wave is two-dimensional. When this two-dimensional wave is linearly amplified to sufficiently high amplitude, nonlinear interaction comes into play through a sequence of secondary instabilities [7]. Besides, as the oblique second-mode at small wave angles are almost as amplified as two-dimensional waves, the oblique-mode breakdown is also a viable path to turbulence with initially only a pair of oblique waves $[8,9]$. Many numerical and experimental researches were conducted on the cone boundary-layers with free-stream Mach numbers of 6 and $8[8,10-12]$. Their results indicated that the fundamental and oblique-mode breakdown were the two most possible transition mechanisms. Furthermore, Chen et al. [13] revealed the important role of the combination resonance where the low-frequency first and Görtler modes were heavily amplified through the interaction with the second mode.

For high-enthalpy boundary layers, the linear instability characteristics were heavily studied using LST and linear parabolized stability equations (PSE) [14-16]. It was found that the second mode became destabilized with higher frequency as the TCNE boundary layer became cooler and thinner. On the other hand, the relaxation processes and endothermic reactions can have damping effects on the second-mode disturbances depending on the Damkhöther number, which is defined as the time-scale ratio between the flow and the TCNE process. Based on these linear results, the $e^{N}$ method can be applied for the transition prediction [17]. In contrast, the nonlinear stages in high-enthalpy boundary layers received much less attention. Marxen et al. [18] performed the direct numerical simulation (DNS) for the case of the fundamental resonance over a flat-plate of Mach 10. They concluded that chemical reactions indirectly affected the secondary growth rates through the change of the primary instability behaviors. The secondary instabilities were further analyzed by the present authors [19] by using the nonlinear PSE and Floquet analysis. The fundamental resonance was found to dominate over the subharmonic resonance. However, the oblique-mode breakdown in high-enthalpy boundary layers hasn't been explored and its mechanism is still unclear.

This work aims to investigate the oblique-mode breakdown in hypersonic and highenthalpy boundary layers with thermal-chemical non-equilibrium effects. The flow considered is over a blunt cone with zero angle-of-attack and a free-stream Mach-number of 15. The tools of NPSE are employed to study the disturbance evolution and flow structures. The problem formulation is provided in Section 2 and the simulation setup is given in Section 3. Section 4 shows the linear instability analyses and the nonlinear stages are discussed in Section 5. The effects of TCNE are investigated in Section 6, and the work is concluded in Section 7.

\section{Formulations}

\subsection{Governing equations}

At the temperature lower than $9000 \mathrm{~K}$, ionization and electronic processes are usually insignificant. Air can be treated as a mixture of five species $\left(\mathrm{N}_{2}, \mathrm{O}_{2}, \mathrm{NO}, \mathrm{N}, \mathrm{O}\right)$. The governing Navier-Stokes equation for TCNE flows in Cartesian coordinates is written in Eq. 1. It contains extra conservations of species mass and vibrational energy as compared with that for CPG flows. 


$$
\begin{gathered}
\frac{\partial \mathbf{U}}{\partial t}+\frac{\partial \mathbf{F}_{j}}{\partial x_{j}}=\frac{\partial \mathbf{F}_{v, j}}{\partial x_{j}}+\mathbf{S} \\
\mathbf{U}=\left[\begin{array}{c}
\rho \\
\rho u_{i} \\
\rho E \\
\rho_{s} \\
\rho e_{v}
\end{array}\right] \quad \mathbf{F}_{v, j}=\left[\begin{array}{c}
\tau_{i j} \\
u_{k} \tau_{j k}+\kappa \frac{\partial T}{\partial x_{j}}+\kappa_{v} \frac{\partial T_{v}}{\partial x_{j}}-\sum_{m=1}^{5} h_{m} d_{m j} \\
-d_{s j} \\
\kappa_{v} \frac{\partial T_{v}}{\partial x_{j}}-\sum_{m=1}^{3} e_{v, m} d_{m j} \\
0 \\
0 \\
\mathbf{F}_{j}=\left[\begin{array}{c}
\rho u_{j} \\
\rho u_{i} u_{j}+p \delta_{i j} \\
\rho u_{j} H \\
\rho_{s} u_{j} \\
\rho u_{j} e_{v}
\end{array}\right] \mathbf{S}=\left[\begin{array}{c}
\dot{\omega}_{s} \\
3 \\
Q_{t-v}+\sum_{m=1}^{3} e_{v, m} \dot{\omega}_{m}
\end{array}\right]
\end{array}\right.
\end{gathered}
$$

Here the subscript $s \in[2,5]$ is the species index; $\rho, u_{i}, p$ and $E$ respectively density, velocities, pressure and specific total energy. The two-temperature model by Park [20] is employed, which consists of translational/rotational temperature $T$ and vibrational temperature $T_{v}$. The mixture's specific total enthalpy $H$ and vibrational energy $e_{v}$ are weighted by the mass fraction $Y_{s}=\rho_{s} / \rho . \tau_{i j}$ is the viscous stress, while $\kappa$ and $\kappa_{v}$ are the conductivity coefficients. The term $d_{s j}$ stands for the species mass diffusion. The two TCNE source terms, $Q_{t-v}$ and $\dot{\omega}_{s}$, are introduced to describe respectively the translational-vibrational energy relaxation and the finite-rate chemical reactions. The details of the adopted TCNE models can be found in the authors' previous works [21,22].

A laminar mean flow is needed first for stability analyses. For two-dimensional or axisymmetric mean flows, Eq. 1 is solved through a 5th-order shock-fitting solver [22].

\subsection{Parabolized stability analysis}

The implementation of the NPSE analysis is briefly described here with more details found in Ref. [23]. The physical quantity $\boldsymbol{q}$ is decomposed into a steady part $\overline{\boldsymbol{q}}$ and a disturbed part $\widetilde{\boldsymbol{q}} \cdot \overline{\boldsymbol{q}}=\left[\bar{\rho}, \bar{U}, \bar{V}, \bar{W}, \bar{T}, \bar{Y}_{s}, \bar{T}_{\nu}\right](s \in[2,5])$ is the laminar mean-flow solution while $\widetilde{\boldsymbol{q}}=\left[\tilde{\rho}, \tilde{u}, \tilde{v}, \tilde{w}, \tilde{T}, \tilde{y}_{s}, \tilde{T}_{v}\right]$ the disturbance. After substituting the decomposition into Eq. 1, the governing equation for disturbances is written in the cylindrical coordinate $(x-y-\theta)$ as,

$$
\begin{aligned}
\boldsymbol{F} \frac{\partial \widetilde{\boldsymbol{q}}}{\partial t} & +\boldsymbol{A} \frac{\partial \widetilde{\boldsymbol{q}}}{\partial x}+\boldsymbol{B} \frac{\partial \widetilde{\boldsymbol{q}}}{\partial y}+\frac{\boldsymbol{C}}{r} \frac{\partial \widetilde{\boldsymbol{q}}}{\partial \theta}+\boldsymbol{D} \widetilde{\boldsymbol{q}}=\boldsymbol{H}_{x x} \frac{\partial^{2} \widetilde{\boldsymbol{q}}}{\partial x^{2}}+\boldsymbol{H}_{y y} \frac{\partial^{2} \widetilde{\boldsymbol{q}}}{\partial y^{2}} \\
& +\frac{\boldsymbol{H}_{z z}}{r^{2}} \frac{\partial^{2} \widetilde{\boldsymbol{q}}}{\partial \theta^{2}}+\boldsymbol{H}_{x y} \frac{\partial^{2} \widetilde{\boldsymbol{q}}}{\partial x \partial y}+\frac{\boldsymbol{H}_{x z}}{r} \frac{\partial^{2} \widetilde{\boldsymbol{q}}}{\partial x \partial \theta}+\frac{\boldsymbol{H}_{y z}}{r} \frac{\partial^{2} \widetilde{\boldsymbol{q}}}{\partial y \partial \theta}+\boldsymbol{N}
\end{aligned}
$$

where $\boldsymbol{F}, \boldsymbol{A}, \boldsymbol{B}, \boldsymbol{C}, \boldsymbol{D}$ and $\boldsymbol{H}$ are all $10 \times 10$ matrices, related only to $\overline{\boldsymbol{q}}$ and $\boldsymbol{N}$ represents non-linear terms; $r=r(x, y)$ the coordinate radius. The following Fourier decomposition is introduced for the disturbance:

$$
\tilde{\boldsymbol{q}}(x, y, \theta, t)=\sum_{m, n} \hat{\boldsymbol{q}}_{m n}(x, y) \exp \left[\mathrm{i}\left(\int_{x_{0}}^{x} \alpha_{m n}(\bar{x}) \mathrm{d} \bar{x}+n k_{c} \theta-m \omega t\right)\right]
$$

where $\omega$ and $k_{c}$ are respectively the specified minimum circular frequency and azimuthal wavenumber. Here $k_{c}$ is related to the azimuthal wavelength through $\lambda_{\theta}=2 \pi r / k_{c} . \hat{\boldsymbol{q}}_{m n}$ is the disturbance shape function and $\alpha_{m n}=\alpha_{m n, r}+\mathrm{i} \alpha_{m n, i}$ the complex streamwise 
wavenumber. Phase speed is defined as $c_{m n, r}=m \omega / \alpha_{m n, r}$. As a result, the parabolized stability equation for each component is written as:

$$
\hat{\mathbf{D}}_{m n} \hat{\boldsymbol{q}}_{m n}+\hat{\mathbf{A}}_{m n} \frac{\partial \hat{\boldsymbol{q}}_{m n}}{\partial x}+\hat{\mathbf{B}}_{m n} \frac{\partial \hat{\boldsymbol{q}}_{m n}}{\partial y}+\hat{\mathbf{C}}_{m n} \frac{\partial^{2} \hat{\boldsymbol{q}}_{m n}}{\partial y^{2}}=\hat{\boldsymbol{N}}_{m n} \exp \left(-\mathrm{i} \int_{x_{0}}^{x} \alpha_{m n} \mathrm{~d} x\right)
$$

The wall-normal discretization employs the Chebyshev collocation point method and the streamwise one adopts a backward Euler scheme.

For boundary conditions, the disturbance at the wall satisfies

$$
\hat{u}_{m n}=\hat{v}_{m n}=\hat{w}_{m n}=\hat{T}_{m n}=\hat{T}_{v, m n}=\hat{y}_{s, m n}=0 \quad \text { at } \quad y=0
$$

Nevertheless, for steady modes the wall boundary conditions take the same form as those for laminar flows (no-slip, isothermal and non-catalytic). In the far-field, the boundary is coupled with the shock oscillation to account for shock-disturbance interactions [24]. Verification of the present solver is provided in Ref. [19].

\section{Laminar flow field}

The geometry considered is a blunt cone with a nose radius of $5 \mathrm{~mm}$ and a half-angle of $7^{\circ}$. The free-stream conditions are listed in Table 1, which correspond to the altitude of $25 \mathrm{~km}$. The wall temperature is fixed at $1000 \mathrm{~K}$ over most of the cone surface. However, a radiative wall-boundary condition is employed on the nose sphere region to allow higher wall temperature because of the high local heat fluxes [25].

The laminar mean flow is depicted in Fig. 1. The high free-stream Mach-number leads to a thin shock layer. The distance between the shock and the wall is only $55 \mathrm{~mm}$ at $x$ of $2 \mathrm{~m}$. Figure $1(b)$ provides a close-up view of the flow around the nose region. Owing to the extremely high temperature behind the bow shock, the vibrational energy is highly excited and the oxygen is almost dissociated near the wall. In the following, the local surface coordinate $(s-y-\theta)$ is used for convenience with the origin of $s$ at the stagnation point, as sketched in the figure.

The boundary layer profiles are shown in Fig. 2 at two streamwise locations of $0.4 \mathrm{~m}$ and $1.6 \mathrm{~m}$. The pressure in the wall-normal direction remains almost constant across the boundary layer and slightly decreases outside. The maximum temperature in the boundary layer is $2790 \mathrm{~K}$ at $s$ of $0.4 \mathrm{~m}$ and falls to $2130 \mathrm{~K}$ at $1.6 \mathrm{~m}$. The gaps between $T$ and $T_{v}$ are around $700 \mathrm{~K}$, indicating that the flow is in thermal non-equilibrium. From the species fraction profiles, the flow tends to be chemical equilibrium further downstream. At the location of $1.6 \mathrm{~m}$, the maximum mass fractions of $\mathrm{NO}$ and $\mathrm{O}$ are less than $0.5 \%$.

\section{Linear stability results}

The linear instability behaviors are analyzed first using LST to determine the parameters for nonlinear calculations. Figure 3(a) gives the neutral curves for axisymmetric disturbance waves. Owing to the relatively low wall temperature, the first mode instability is not observed within the computational domain. The second mode has the highest growth rate

Table 1 Free-stream conditions for the Mach 15 flow over a blunt cone

\begin{tabular}{lllllll}
\hline$M_{\infty}$ & $p_{\infty}(\mathrm{Pa})$ & $T_{\infty}(\mathrm{K})$ & $U_{\infty}(\mathrm{m} / \mathrm{s})$ & $\boldsymbol{R e}_{\infty}(/ \mathrm{m})$ & $Y_{\mathrm{N}_{2}, \infty}$ & $T_{\mathrm{w}}(\mathrm{K})$ \\
\hline 15 & 2549.2 & 221.55 & 4475.8 & $1.265 \times 10^{7}$ & 0.767 & 1000 \\
\hline
\end{tabular}




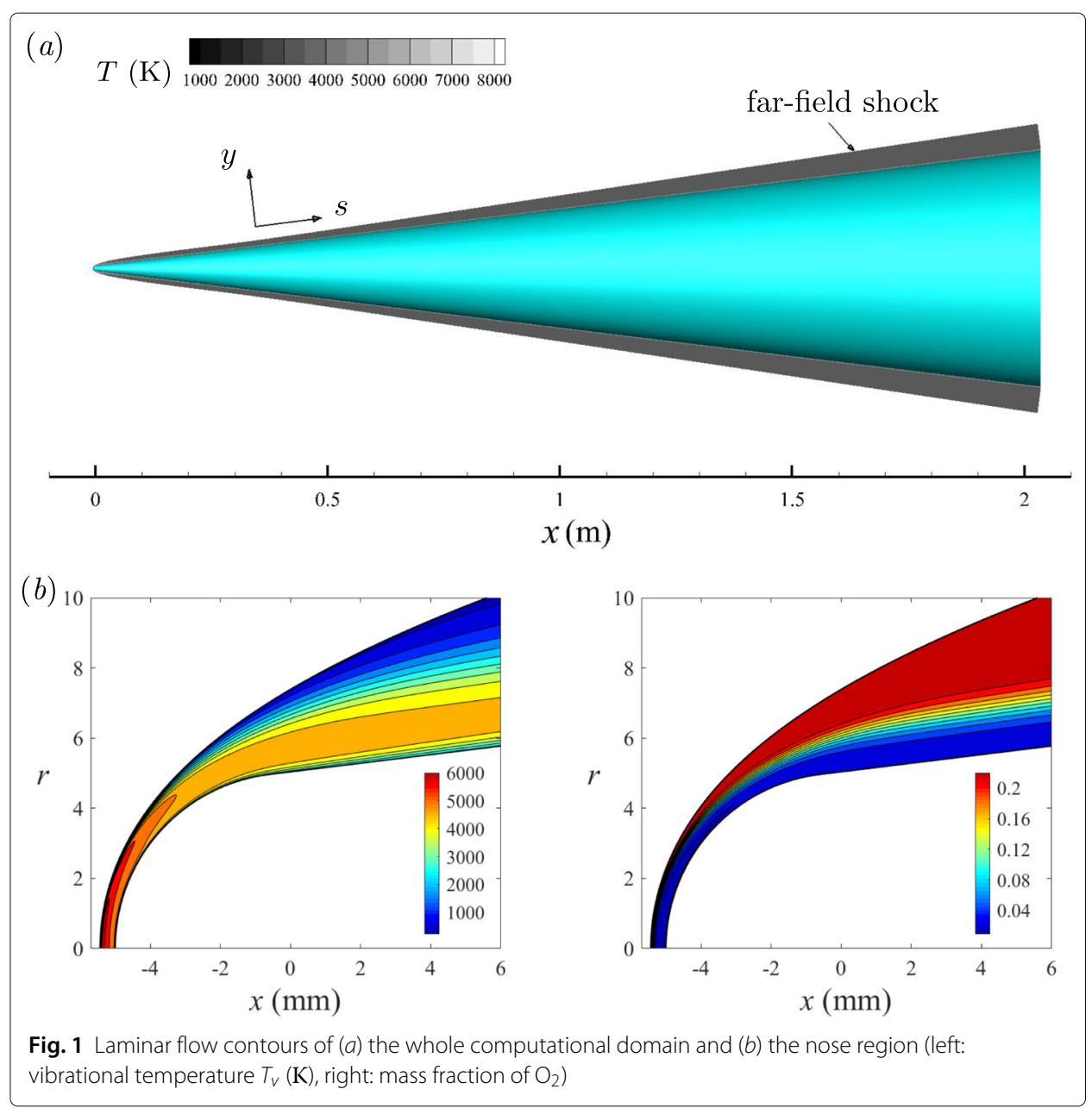

with a frequency range of $310 \mathrm{kHz}$ to $750 \mathrm{kHz}$ down to $s=2 \mathrm{~m}$. In contrast, the instability region of the third mode appears further downstream and at a higher frequency range of $780 \mathrm{kHz}$ to $1080 \mathrm{kHz}$. The variation of the growth rate with the azimuthal wavenumber $k_{c}$ is shown in Fig. $3(b)$ at $s=1.6 \mathrm{~m}$. The second mode is most unstable when axisymmetric, and becomes stable at $k_{c}$ larger than 237. In contrast, the most unstable first mode is three-dimensional. The instability region of the first mode appears at $k_{c}$ ranging from 44 to 117 , but its growth rate is much smaller as compared with the second mode.

The eigenvectors of the axisymmetric and oblique second-mode disturbances are displayed in Fig. 4 with the frequency of $500 \mathrm{kHz}$ at $s$ of $1 \mathrm{~m}$. Here all the curves are normalized based on the pressure disturbance at the wall. The three selected azimuthal wavenumbers are 0,75 and 150, respectively. The corresponding wave angles $\psi=$ $\arctan (\beta / \alpha)$ (where $\beta=k_{c} / r$ ) for the latter two waves are $39.0^{\circ}$ and $58.8^{\circ}$. Because of the high free-stream Mach-number, the amplitude of the temperature disturbance $\left|\hat{T} / T_{\infty}\right|$ is nearly 20 times the velocity disturbance. The disturbance near the critical layer (where $\bar{U}=c_{r}$ ) is mostly affected by $k_{c}$ due to inviscid singularity [6]. With the increase of $k_{c}$, peaks appear around the critical layer for all the quantities in the figure except for $\hat{p}$ and $\hat{v}$. The shapes of the latter two are nearly unchanged across the boundary layer.

The $N$ factors for axisymmetric waves are plotted in Fig. 5 with different frequencies. The contributions to the $N$ factors here are from the second-mode waves. For the curve 

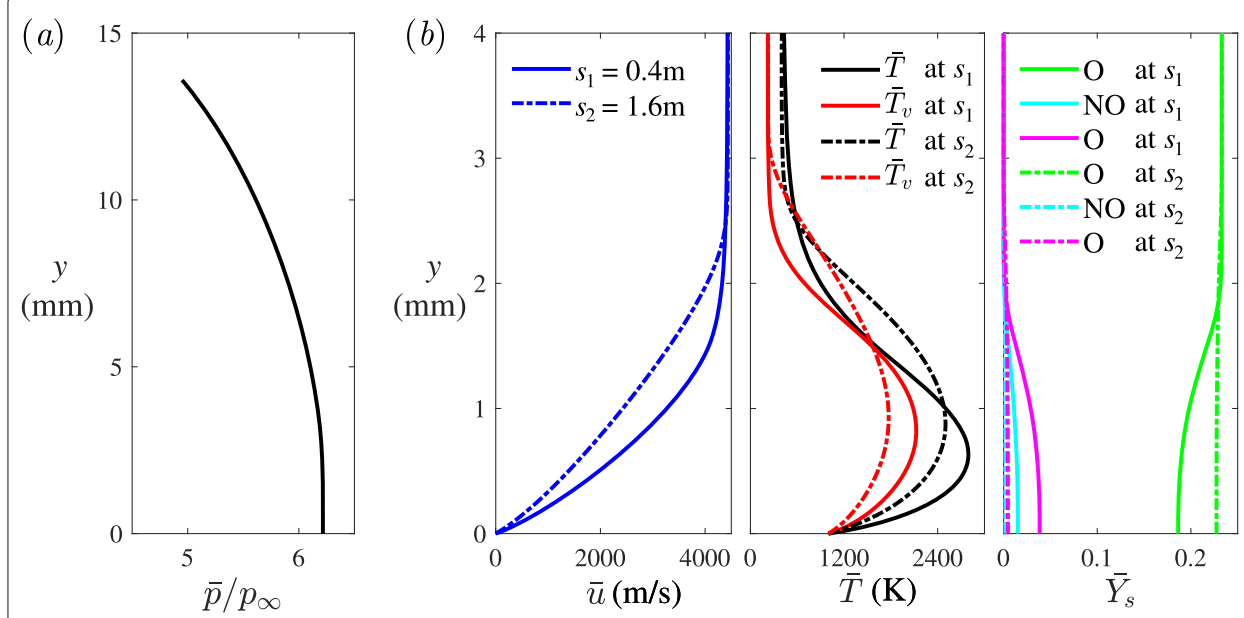

Fig. 2 Laminar boundary-layer profiles of $(a)$ the pressure at $s=0.4 \mathrm{~m},(b)$ the streamwise velocity, temperature and mass fraction at $s$ of $0.4 \mathrm{~m}$ and $1.6 \mathrm{~m}$
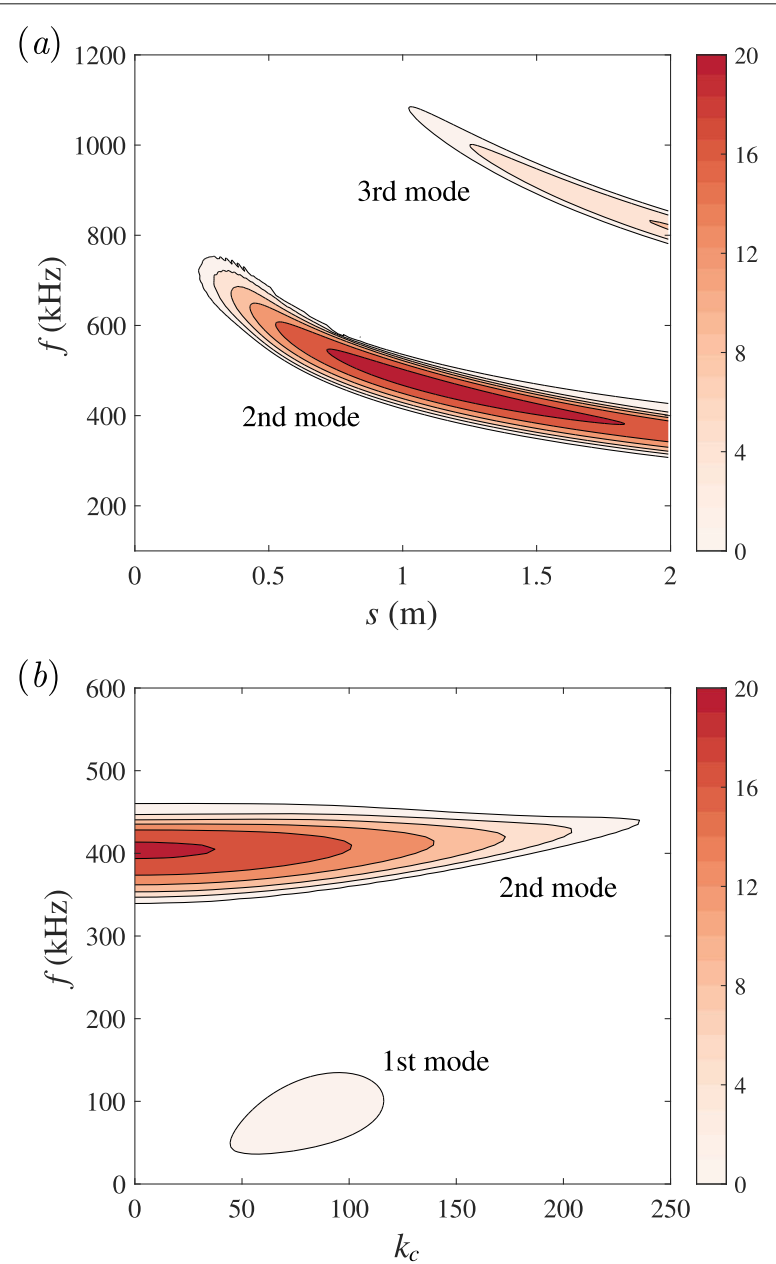

Fig. 3 Growth rate contours $\left(-\alpha_{i}, 1 / \mathrm{m}\right)(a)$ for axisymmetric waves and (b) with the azimuthal wavenumber at $\mathrm{s}=1.6 \mathrm{~m}$ 


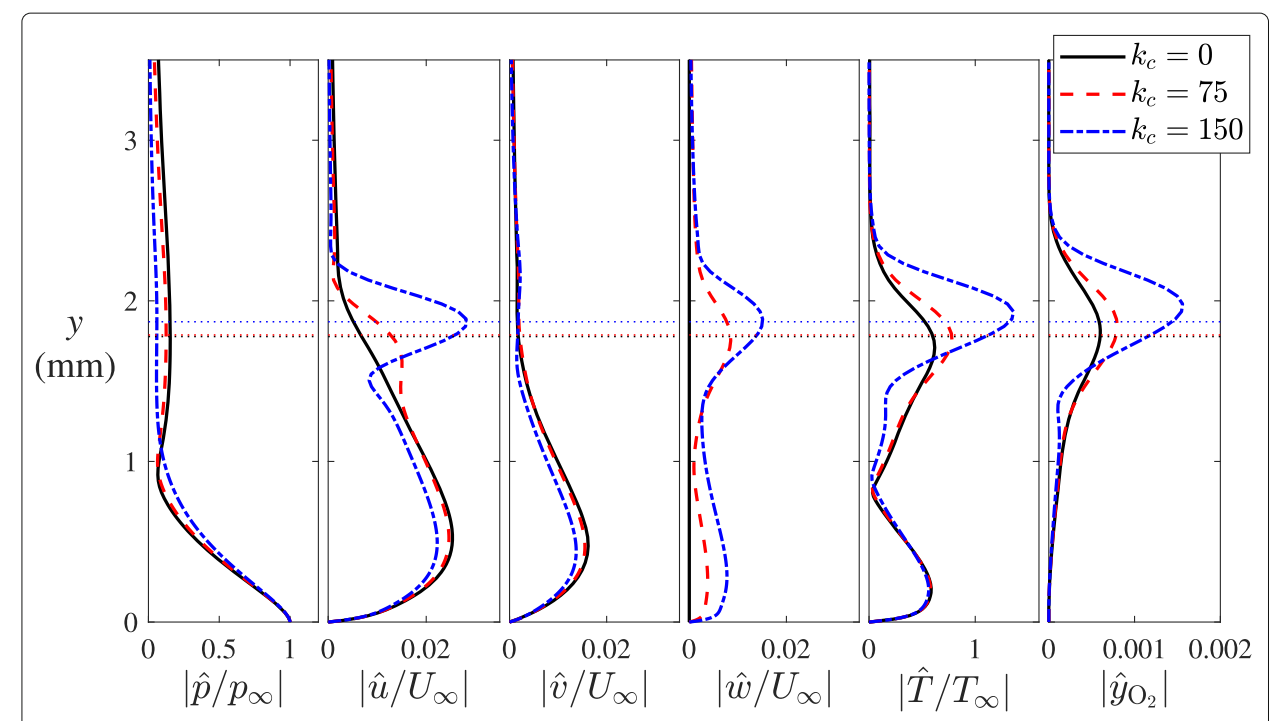

Fig. 4 Eigenvectors of the second-mode disturbance with different azimuthal wavenumbers at $s$ of $1 \mathrm{~m}$. The disturbance frequency is $500 \mathrm{kHz}$. The subfigures are the disturbance curves of (a) pressure, $(b-d)$ velocities, (e) temperature and ( $f$ ) mass fraction of $\mathrm{O}_{2}$

with $f=400 \mathrm{kHz}$, the maximum $N$ factor is over 14 . The transition onset location can be determined from the $e^{N}$ method after a correlation to experimental results.

\section{Nonlinear results}

\subsection{Oblique-wave parameters}

The oblique-mode breakdown is initiated by a symmetric pair of oblique waves $(1, \pm 1)$. Here the notation $(m, n)$ represents the wave with the frequency of $m f$ and the azimuthal wavenumber of $n k_{c}$. The oblique-wave parameters $f$ and $k_{c}$ need to be determined first.

The oblique-wave frequency is determined from the $N$ factor curves in Fig. 5. Malik [26] provided an estimation of the transition $N$ factor to be between 9.5 to 12 based on the flight data with free-stream Mach-numbers of over 20. The experiments in the HIEST facility gave a value of 8 [27]. Therefore, the disturbance frequency $f$ is selected to be $500 \mathrm{kHz}$, which corresponds to a maximum $N$ factor of around 9.

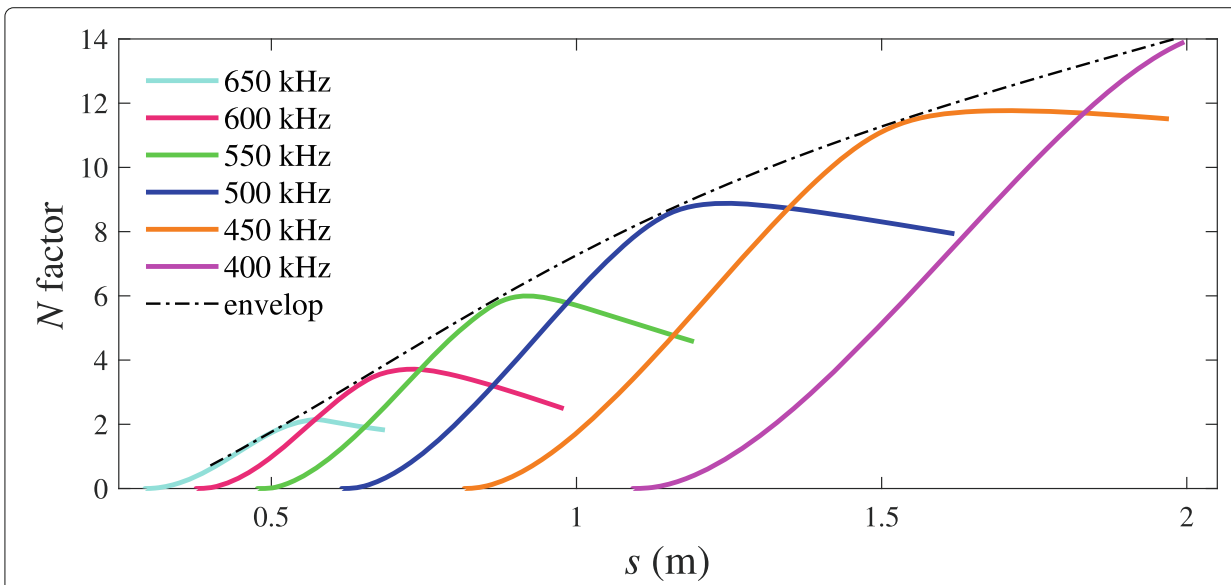

Fig. $5 \mathrm{~N}$ factor curves of axisymmetric second-mode waves for the disturbances with different frequencies 
The azimuthal wavenumber is obtained through a parameter study. At $k_{c}=10$, the streamwise development of representative waves is displayed in Fig. 6(a). Here the amplitudes of different modes are measured by the root mean square of the streamwise-velocity disturbance $\tilde{u}_{\text {rms }}$, normalized by the free-stream velocity $U_{\infty}$. The initial amplitude $A_{(1,1)}$ is $0.05 \%$. The linear evolution of mode $(1,1)$ is also plotted for reference. As can be seen, mode $(1,1)$ in the nonlinear calculation follows the linear growth downstream until it reaches the amplitude of $O(0.1)$ at $s$ of $0.98 \mathrm{~m}$ and begins to decay. The axisymmetric wave $(2,0)$ and the streamwise-vortex mode $(0,2)$ are generated through the interaction between $(1, \pm 1)$. Mode $(0,2)$ exceeds $(1,1)$ in amplitude at $s$ of $1 \mathrm{~m}$ to become the dominant one. Zhang [28] showed that this rapid growth of the streamwise-vortex mode was determined by the characteristics of the governing linear operator. Mode $(0,4)$ also experiences quick amplification, whose amplitude increases by over 8 orders of magnitude in the figure.

A series of simulations are performed for a range of $k_{c}$ from 5 to 30 in increments of $\Delta k_{c}=5$. The amplitudes of modes $(1,1),(0,2)$ and $(0,4)$ are compared in Fig. $6(b)$ at $s=1.02 \mathrm{~m}$. For convenience in comparison, the amplitudes are normalized using the ones at $k_{c}=5$. The amplitude ratio of mode $(1,1)$ decreases with $k_{c}$, which is consistent with that in Fig. 3. For the two steady modes, however, the changes are not monotonous. The case of $k_{c}=20$ has the largest amplitudes of both $(0,2)$ and $(0,4)$. Therefore, the $k_{c}$ of 20 is selected for further calculations. Note that at $k_{c}$ of 20 , the azimuthal wavenumbers for the two steady modes are 40 and 80 . This range matches the azimuthal wavenumber of the most unstable first mode in Fig. 3 with consideration of the body divergence of the cone geometry. This body divergence leads to the increase of $k_{c}$ with $s$ at a fixed wave angle $\psi$.

\subsection{Disturbance evolution and flow structures}

The streamwise development of multiple waves is plotted in Fig. 7(a) with initial modes $(1, \pm 1)$. The waves with higher frequencies and azimuthal wavenumbers are generated in sequence and quickly amplified through nonlinear interactions. The amplitudes of all the waves displayed exceeds $10^{-3}$ at $s$ of $0.99 \mathrm{~m}$. Further downstream, the three streamwise-vortex modes all have larger amplitudes than mode $(1,1)$. To determine the state of the flow, the development of the averaged skin friction is shown in Fig. $7(b)$.
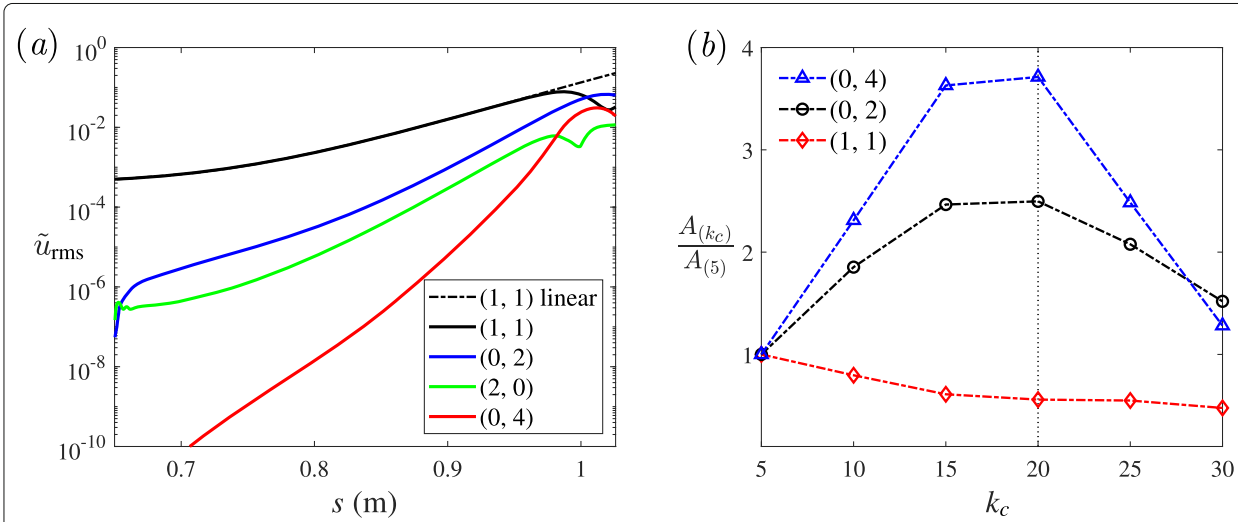

Fig. 6 Streamwise development of representative waves in the oblique-mode breakdown with $f=500 \mathrm{kHz}$ : (a) wave amplitudes with $k_{c}=10$ and $(b)$ amplitude ratios at $s=1.02 \mathrm{~m}$ with $k_{c}$ variation 


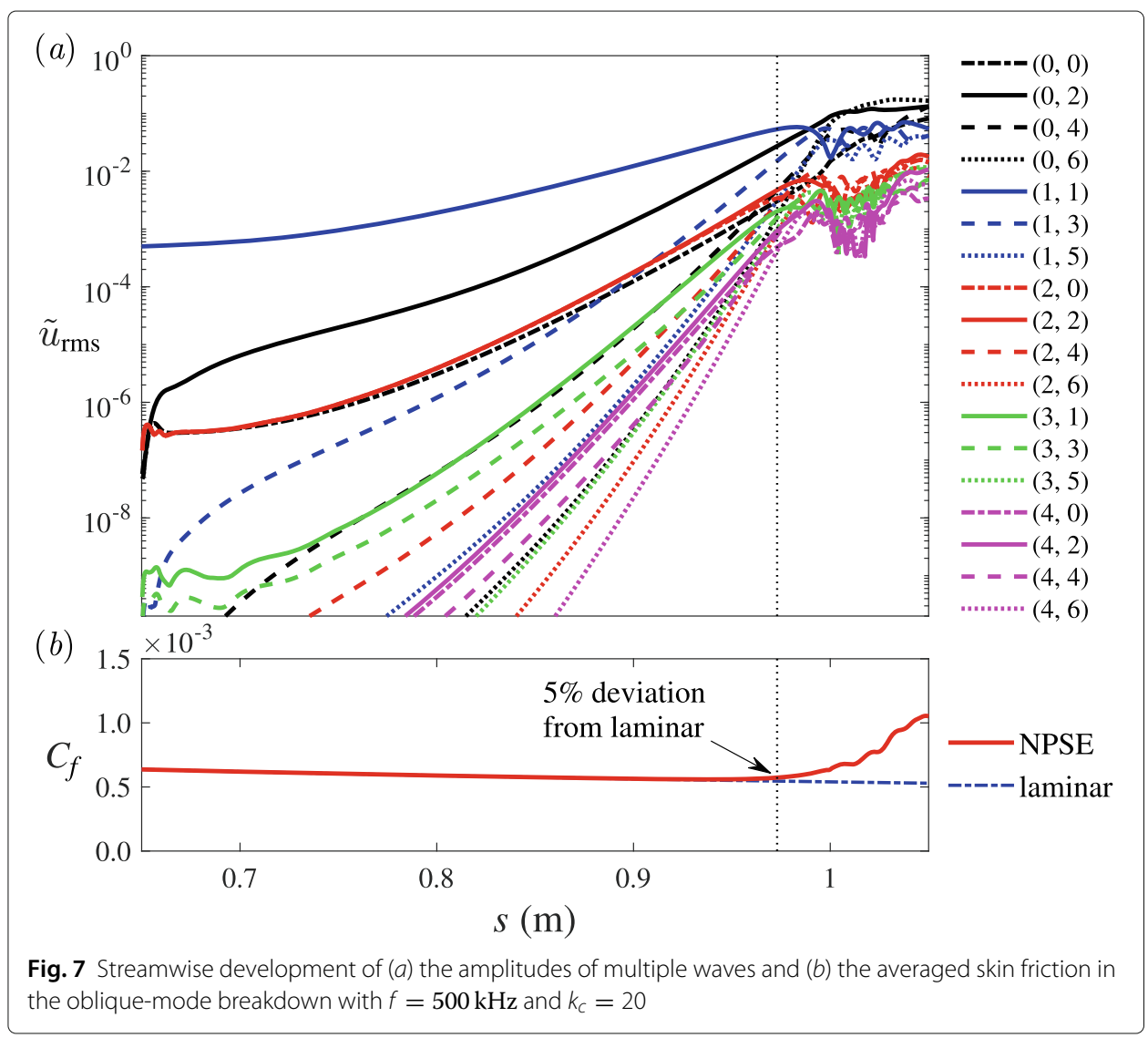

Here the Reynolds-averaged (time- and azimuthal-averaged) skin friction coefficient $C_{f}$ is calculated as

$$
C_{f}=\frac{\left\langle\left.\mu \frac{\partial u}{\partial y}\right|_{y=0}\right\rangle}{\frac{1}{2} \rho_{\infty} U_{\infty}^{2}}
$$

where $\langle\cdot\rangle$ denotes the Reynolds-average operator. The onset of transition is defined here as the location where $C_{f}$ reaches $5 \%$ deviation from the laminar value, which is evaluated to be $0.973 \mathrm{~m}$. This transition onset is exactly the location where mode $(1,1)$ departs from its linear trace. This correlation was also reported in the study on the fundamental breakdown $[10,19]$.

To study the role of the streamwise-vortex modes, the flow is time-averaged to rule out unsteady modes. Figure 8 (a) depicts the contours of the averaged streamwise velocity within one azimuthal period of mode $(1,1)$. The disturbance streamlines in the $y-\theta$ plane show pairs of counter-rotating streamwise vortices. These vortices promote the exchange of mass, momentum and energy between the fluids with low and high speeds [23]. The wall-normal gradient of the temperature at the wall is plotted in Fig. 8(b). This quantity is directly related to the local heat flux. Upstream $s$ of $0.98 \mathrm{~m}$, the heat flux is uniform in the azimuthal direction under the contour levels. Further downstream, six strips are visible with high heat-fluxes. The highest heat-flux appears in the region at around $k_{c}$ of $4.5^{\circ}$ and $13.5^{\circ}$, where the high-speed fluids are heavily driven towards the wall owing to the streamwise vortices. 


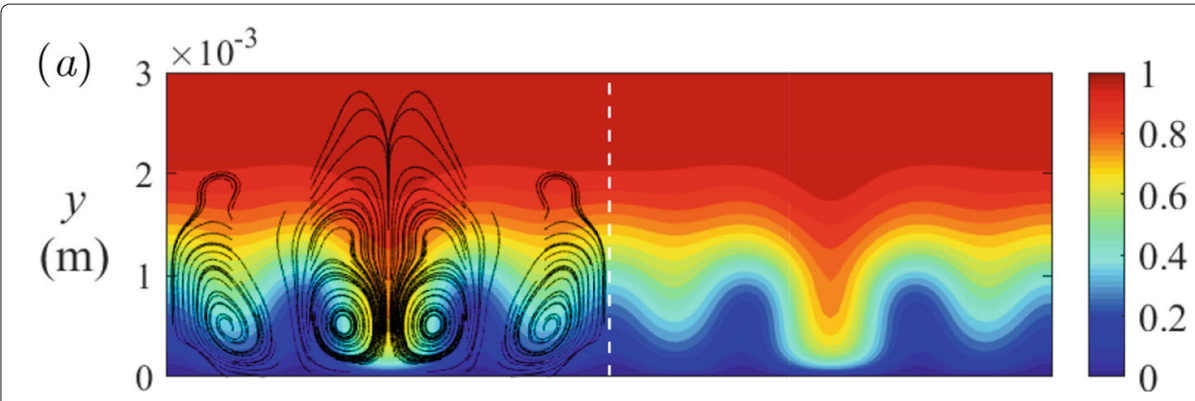

(b)

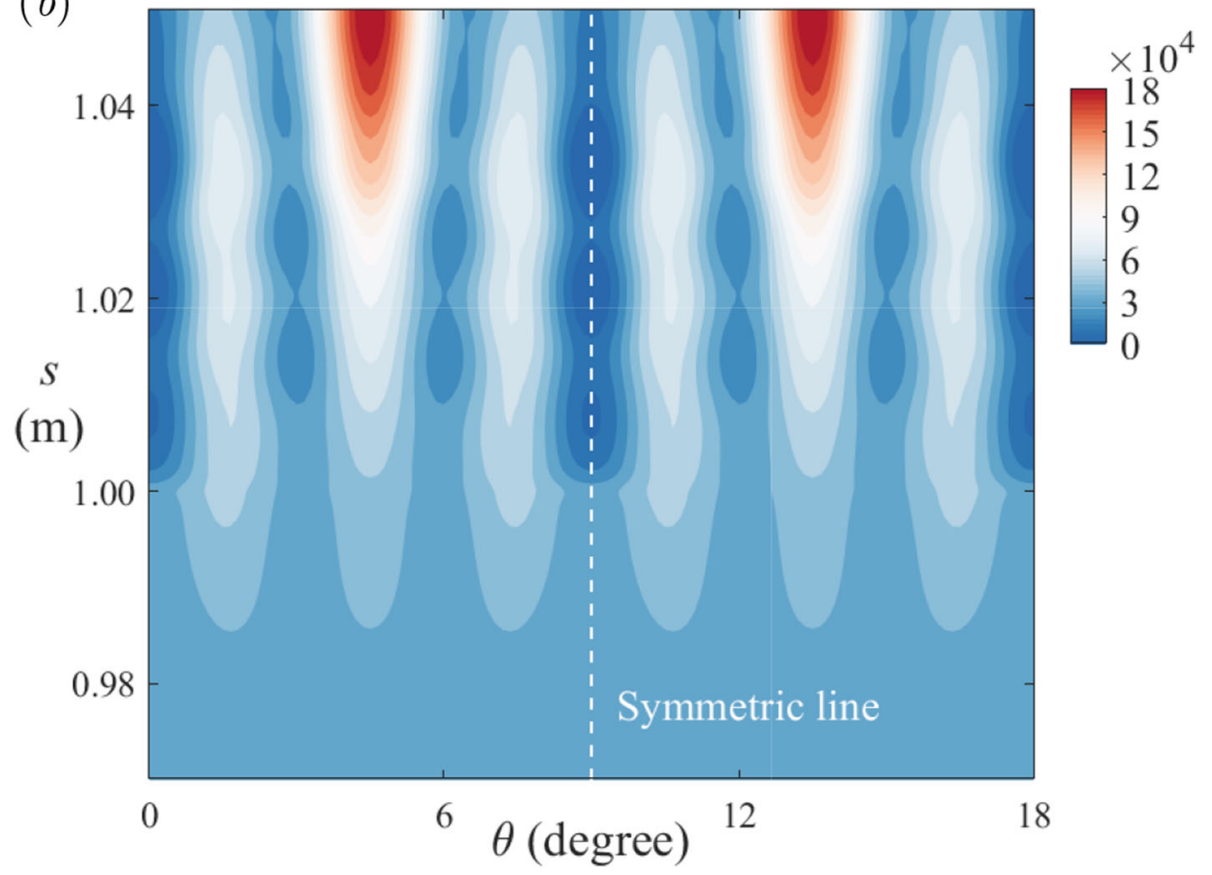

Fig. 8 Contours of the time-averaged flow: (a) the streamwise velocity $\bar{u} / U_{\infty}$ at $s=1.05 \mathrm{~m}$ with the black lines the disturbance streamlines in the $y-\theta$ plane and $(b)$ the wall-normal gradient $\partial \bar{T} /\left(T_{\infty} \partial y\right)$ at the wall

Figure $9(a)$ depicts the contours of the instantaneous streamwise velocity in the $s-\theta$ plane near the critical layer. In the upstream region, the contours are in the staggered pattern resulting from the initial oblique waves. Further downstream four high-speed streaks are visible, as that in Fig. 8. Between the streaks are the low-speed regions whose shape evolves from "dumbbells" into "swallow". The wall-normal gradient of the density is displayed in Fig. $9(b)$ to give a schlieren-like picture. The typical "rope-like" structures are observed near the boundary-layer edge, as revealed in the previous experimental and numerical researches $[8,29]$. These structures are associated with the second mode because their wavelengths are nearly equal to that of mode $(1,1)\left(\lambda_{x} \approx 8 \mathrm{~mm}\right)$. They are distorted downstream $s$ of $1 \mathrm{~m}$ with the amplification of other waves. Meanwhile, acoustic waves are spontaneously radiated away from the wall, which is owing to the synchronization between the second mode and the slow acoustic branch [30].

The shape functions $\hat{T}_{v}$ and $\hat{Y}_{s}$ are further analyzed in this high-enthalpy flow. The results in Refs. $[21,31]$ showed that the linear disturbance was more thermal-chemical 


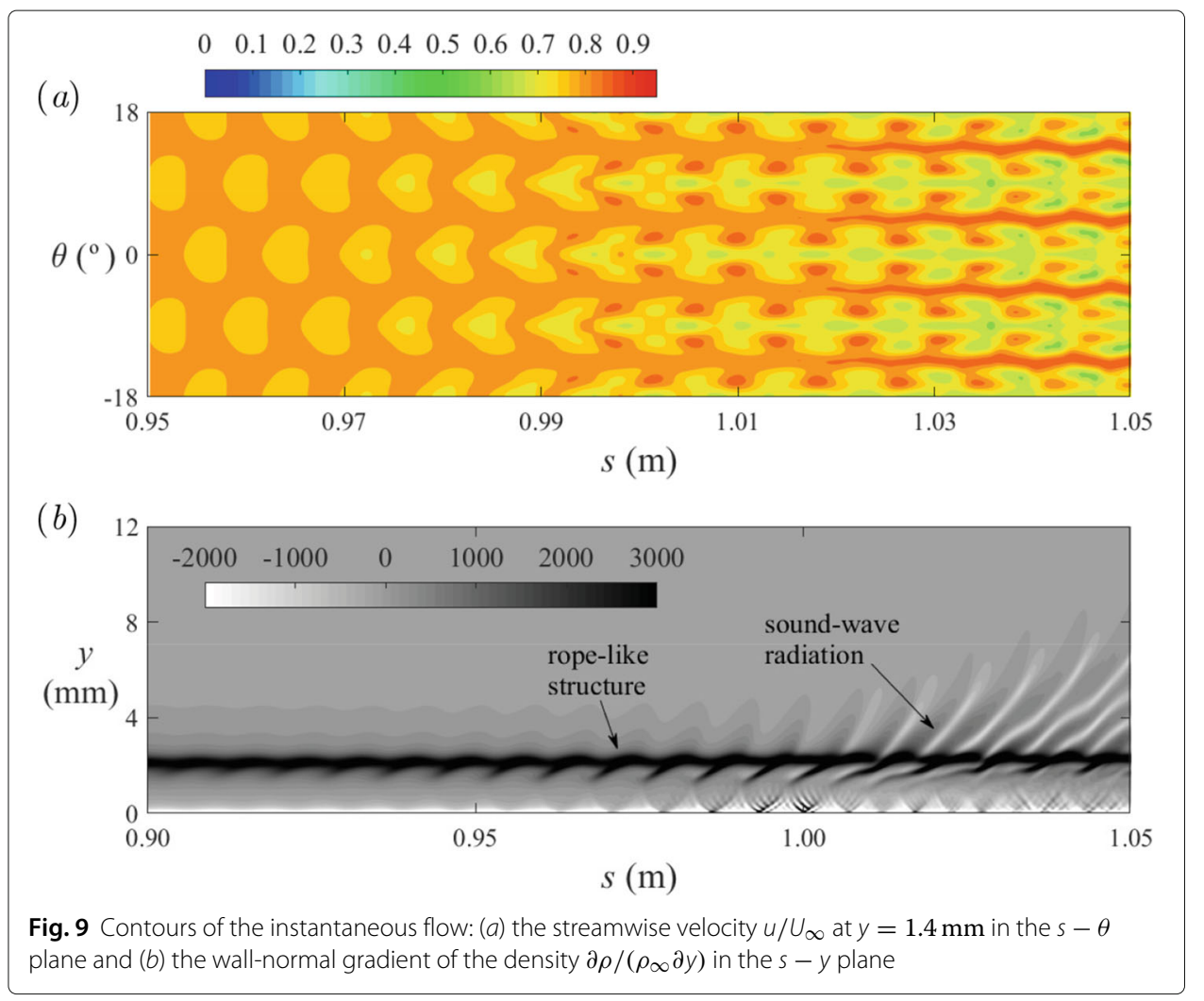

frozen than the mean flow. As a result, the approximate relations of $\hat{T}_{v}$ and $\hat{Y}_{s}$ were provided:

$$
\hat{T}_{v} \approx \frac{\mathrm{i}\left(\mathrm{D}_{y} \bar{T}_{v}\right) \hat{v}}{2 \pi(\bar{U} / c-1) f}, \quad \hat{Y}_{s} \approx \frac{\mathrm{i}\left(\mathrm{D}_{y} \bar{Y}_{s}\right) \hat{v}}{2 \pi(\bar{U} / c-1) f}
$$

Here $\mathrm{D}_{y}=\mathrm{d} / \mathrm{d} y$. This relation is derived from the inviscid Rayleigh equation and by neglecting the disturbances of the TCNE source terms. It shows that $\hat{T}_{v}$ and $\hat{Y}_{s}$ are mainly generated by the wall-normal velocity disturbance $\hat{v}$ along with the wall-normal gradient of the mean flow. The approximation of Eq. 7 for mode $(1,1)$ is verified in Fig. 10, where the two selected locations are in the linear $(s=0.90 \mathrm{~m})$ and nonlinear $(s=1.03 \mathrm{~m})$ stages, respectively. At $s=0.90 \mathrm{~m}$, the shape functions $\hat{T}_{v}$ and $\hat{Y}_{s}$ from NPSE are wellapproximated by the simple relations of Eq. 7 . The only differences are around the critical layer where the denominators of Eq. 7 tend to zero. Going downstream into the nonlinear region, the near-wall structures in Fig. 10(b) are still well-approximated. However, the peaks in NPSE are lifted higher than the critical layer, which is associated with the increase of the boundary layer thickness. This indicates that the near-wall structures of $\hat{T}_{v}$ and $\hat{Y}_{s}$ are still dominated by the convective process as described in Eq. 7, while the disturbances near the boundary layer edge are mainly influenced by the nonlinear interactions.

\section{Effects of thermal-chemical non-equilibrium}

For comparison, the laminar flow and instability results are also calculated within the CPG assumption. The same air composition and transport models (instead of Sutherland's law) are employed for consistency. As is shown in Fig. 11, the second mode is destabilized and the frequency corresponding to the most unstable one increases in the 

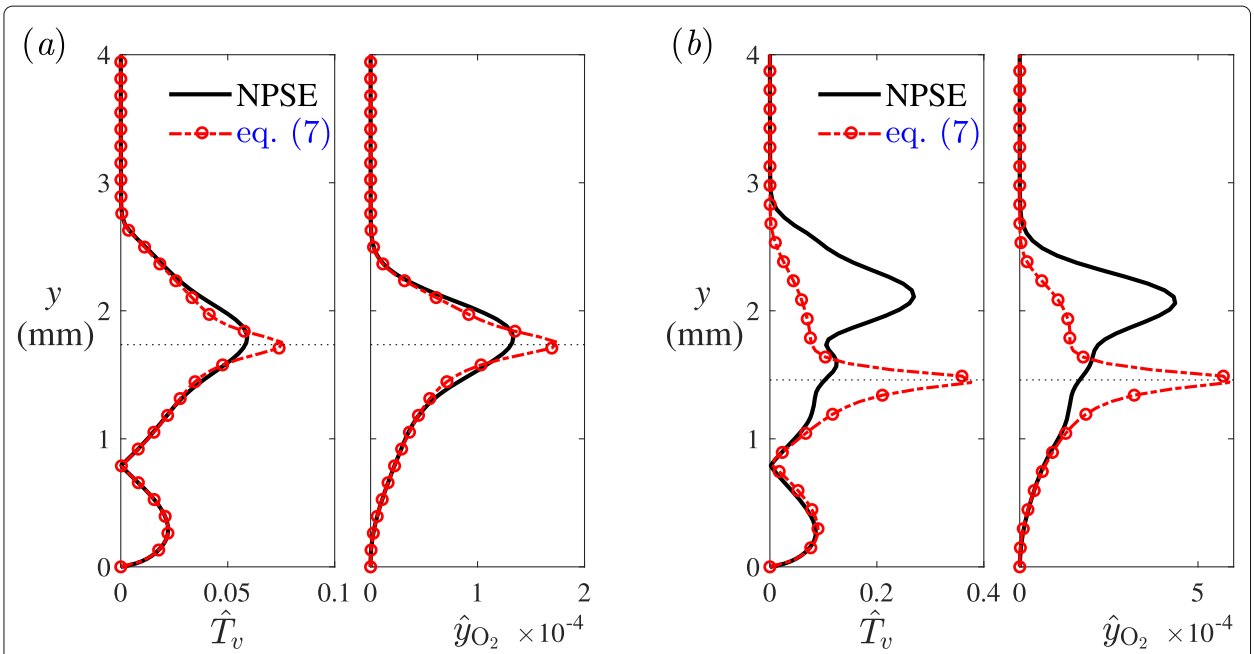

Fig. 10 Eigenvectors of mode $(1,1)$ given by NPSE and approximated by Eq. 7 at $(a) s=0.90 \mathrm{~m}$ and (b) $\mathrm{s}=1.03 \mathrm{~m}$

TCNE flow as the boundary layer becomes cooler and thinner. Therefore, the maximum growth rate in the TCNE case is $16 \%$ higher with $f=500 \mathrm{kHz}$ and the peak location is shifted downstream. Seen from Fig. 11(c), the differences between the maximum $N$ factors at these frequencies are ranging from 1.3 to 2.4. As a result, the envelop of the $N$ factors in the TCNE flow moves upstream in the regime from $0.14 \mathrm{~m}$ to $0.25 \mathrm{~m}$.
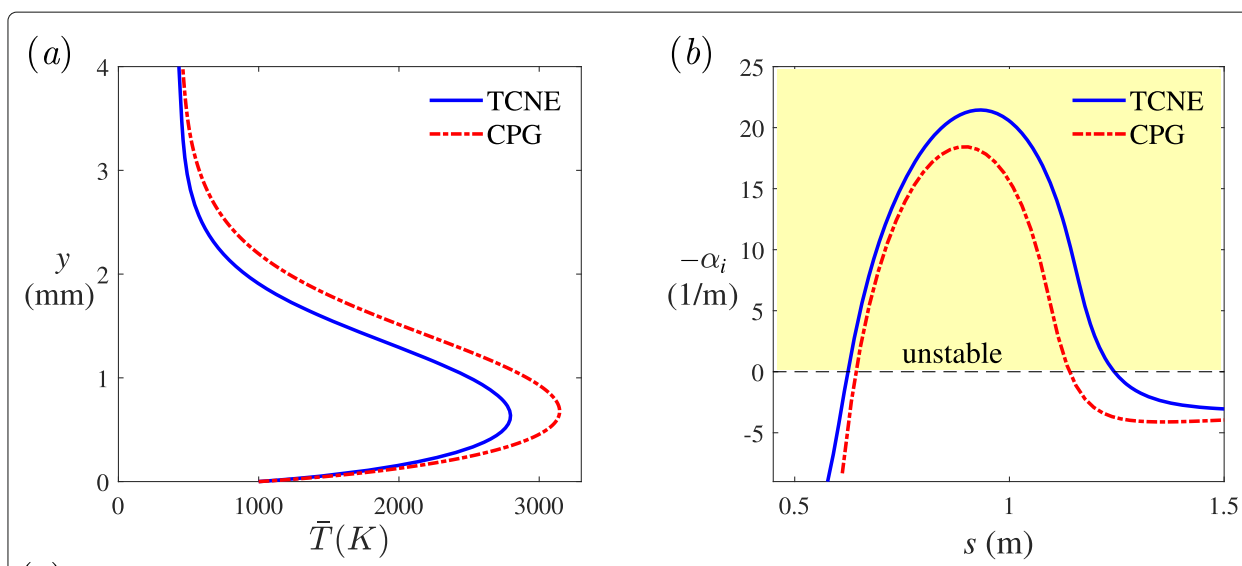

(c)

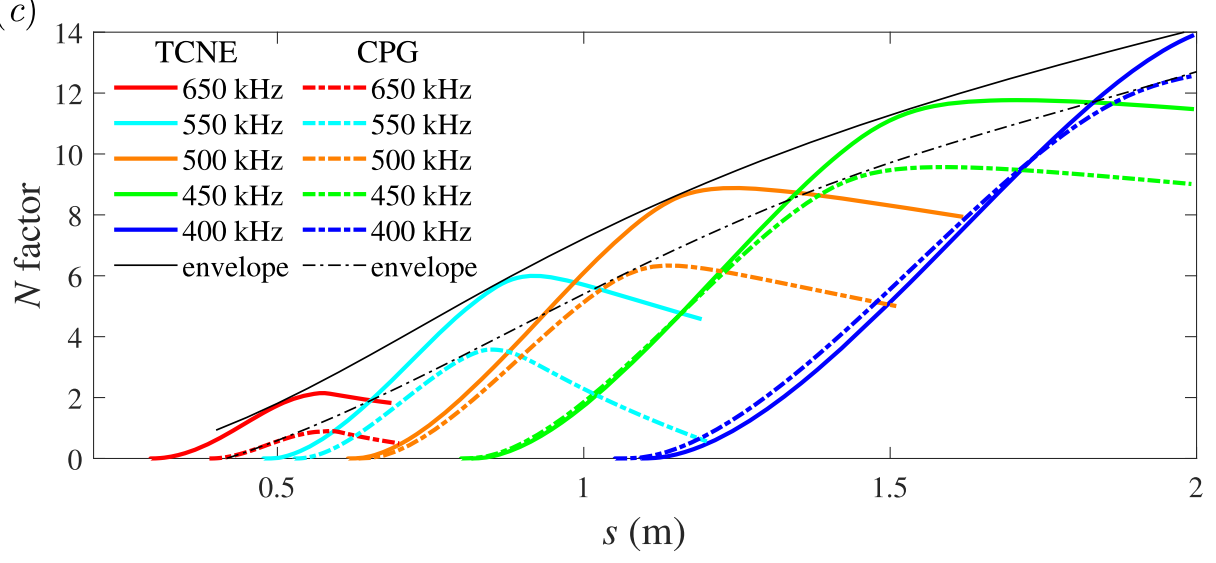

Fig. 11 Comparisons of (a) the laminar flow profile at $s=0.4 \mathrm{~m},(b)$ the growth rate curves with $f$ of $500 \mathrm{kHz}$ and (c) the N factor curves between the TCNE and CPG cases 


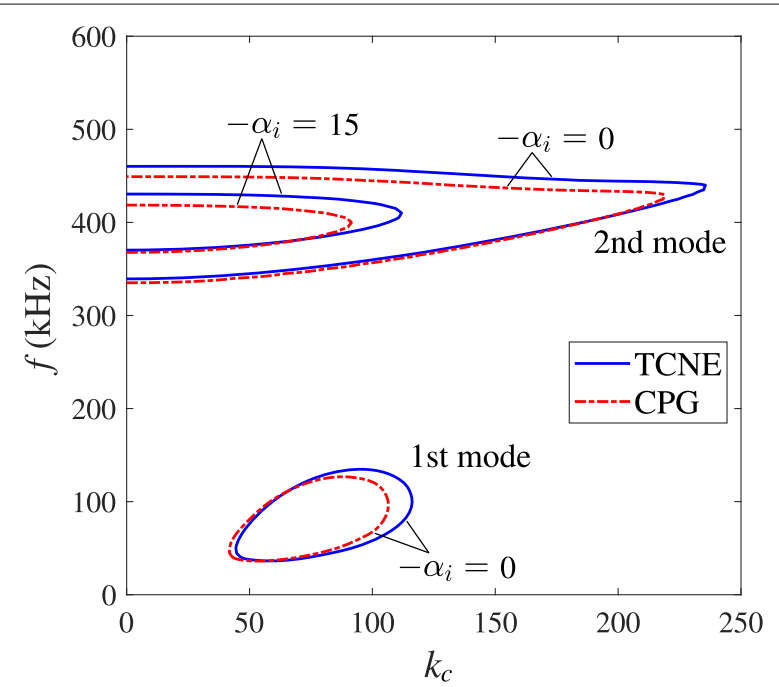

Fig. 12 Growth rate contours $\left(-\alpha_{i}, 1 / \mathrm{m}\right)$ with the azimuthal wavenumber for the TCNE and CPG cases at $s=1.6 \mathrm{~m}$

The effects of TCNE on the three-dimensional waves are shown in Fig. 12 at $s=$ $1.6 \mathrm{~m}$ [see Fig. 3]. The regions of the unstable first and second modes both enlarge in the TCNE case in the frequency-azimuthal-wavenumber plane. As a result, the optimal oblique-wave parameters, as is evaluated in Section 5.1, are different in the CPG case. For comparison, the same parameters as those in the TCNE case are adopted, with $f=500 \mathrm{kHz}$ and $k_{c}=20$ for mode $(1,1)$, to operate the oblique-breakdown calculation for CPG. The comparisons of the streamwise evolution of the representative waves between the TCNE and CPG cases are displayed in Fig. 13. The growth rate of mode $(1,1)$ in the TCNE case is higher with this frequency, so mode $(1,1)$ reaches its maximum amplitude further upstream. Furthermore, modes $(0,2),(2,0),(0,4)$ all grow faster than

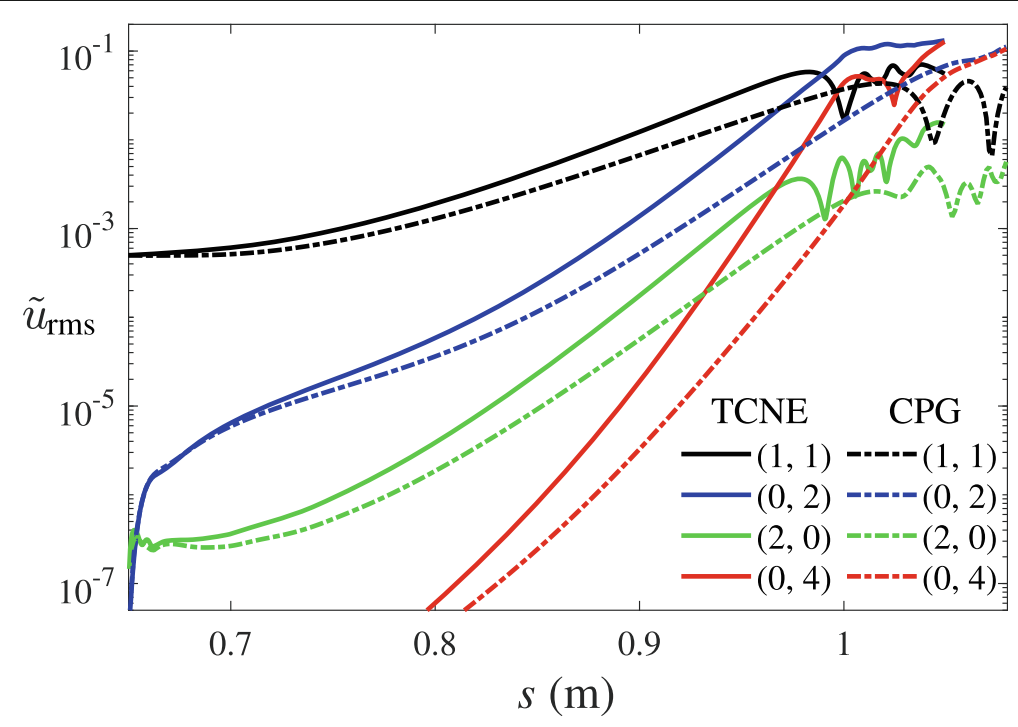

Fig. 13 Streamwise development of representative waves in the oblique-mode breakdown with $f=500 \mathrm{kHz}$ and $k_{c}=20$ for the TCNE and CPG cases 
those in the CPG case to trigger the strong nonlinear interactions because the growth rates of the harmonic waves are strongly related to the amplitude of mode $(1,1)$. Further comparisons of the flow structures indicate that the oblique breakdown process in TCNE flows bears a strong resemblance to that in CPG flows. Nevertheless, the TCNE effects have non-negligible first-order impacts on the waves' growth rates and optimal wave parameters.

\section{Conclusion}

In this work, the oblique-mode breakdown in the hypersonic and high-enthalpy boundary layers is studied using NPSE with consideration of the TCNE effects. The flow over a blunt cone is computed at a free-stream Mach-number of 15 . The frequencies of the oblique second-mode waves are $500 \mathrm{kHz}$ determined from the $N$ factor curves. The azimuthal wavenumber is 20 through a parameter study. It is found that the transition onset (from the skin friction curve) is at the location where mode $(1,1)$ departs from its linear trace, the same as that in the fundamental breakdown. The streamwise-vortex modes have the largest amplitude downstream $s$ of $0.99 \mathrm{~m}$ (see Fig. 7). These vortices lead to the generation of streaks with high local heat fluxes. The rope-like structures and the spontaneous radiation of sound waves are observed in the schlieren-like $(\partial \rho / \partial y)$ picture (see Fig. 9). In the nonlinear stages, the near-wall shapes of mode $(1,1)$ are proportional to the product of the wall-normal velocity disturbance and the mean-flow gradients. The disturbances near the boundary-layer edge are mainly generated through nonlinear interactions. In comparison to the CPG flow, the TCNE effects destabilize the second mode due to cooler and thinner boundary layers. Therefore, the $N$ factor envelope is pushed upstream by $0.14 \mathrm{~m}$ to $0.25 \mathrm{~m}$. The higher growth rate of mode $(1,1)$ leads to stronger growth of the streamwise vortices and harmonic waves, all of which contribute to the strong nonlinear interactions in the transitional regime. The findings in this work can help model the transition physics by focusing on the characteristic flow structures and time-spatial scales of disturbances.

\section{Acknowledgement}

N/A

Authors' contributions

All the authors contributed to and approved this manuscript.

Funding

This work is supported by the National Numerical Wind-tunnel project. The authors also gratefully acknowledge the financial support of the National Key Research and Development Plan of China through the project No. 2019 YFA0405201 and the National Key Project (Grant No. GJXM92579).

Availability of data and materials

The data that support the findings of this study are available from the corresponding author upon reasonable request.

\section{Declarations}

Competing interests

The authors declare that they have no competing interests.

Received: 2 March 2021 Accepted: 10 June 2021

Published online: 10 August 2021

References

1. Chen J, Tu G, Zhang Y, Xu G, Chen C (2017) Hypersonic boundary layer transition: what we know, where shall we go (in Chinese). Acta Aerodynamica Sin 35(3):311-337. https://doi.org/10.7638/kqdlxxb-2017.0030 
2. Shang JS, Yan H (2020) High-enthalpy hypersonic flows. Adv Aerodyn 2(1):19. https://doi.org/10.1186/s42774-02000041-y

3. Gnoffo PA, Gupta RN, Shinn JL (1989) Conservation equations and physical models for hypersonic air flows in thermal and chemical nonequilibrium. NASA Technical Report: NASA-TP-2867. https://ntrs.nasa.gov/citations/19890006744

4. Morkovin MV, Reshotko E, Herbert T (1994) Transition in open flow systems: a reassessment. Bull APS 39(9):1882

5. Reed HL, Saric WS, Arnal D (1996) Linear stability theory applied to boundary layers. Ann Rev Fluid Mech 28:389-428. https://doi.org/10.1146/annurev.fl.28.010196.002133

6. Mack LM (1984) Boundary-layer linear stability theory. https://ntrs.nasa.gov/citations/19840025688

7. Herbert T (1988) Secondary instability of boundary layers. Ann Rev Fluid Mech 20:487-526. https://doi.org/10.1146/ annurev.fluid.20.1.487

8. Pruett CD, Chang C-L (1995) Spatial direct numerical simulation of high-speed boundary-layer flows part II: transition on a cone in Mach 8 flow. Theor Comput Fluid Dyn 7(5):397-424. https://doi.org/10.1007/BF00312416

9. Franko KJ, Lele SK (2013) Breakdown mechanisms and heat transfer overshoot in hypersonic zero pressure gradient boundary layers. J Fluid Mech 730:491-532. https://doi.org/10.1017/jfm.2013.350

10. Sivasubramanian J, Fasel HF (2015) Direct numerical simulation of transition in a sharp cone boundary layer at Mach 6: fundamental breakdown. J Fluid Mech 768:175-218. https://doi.org/10.1017/jfm.2014.678

11. Stetson K, Kimmel R (1993) On the breakdown of a hypersonic laminar boundary layer. In: 31st Aerospace Sciences Meeting. https://doi.org/10.2514/6.1993-896

12. Berridge D, Chou A, Ward C, Steen L, Gilbert P, Juliano T, Schneider S, Gronvall J (2010) Hypersonic boundary-layer transition experiments in a Mach-6 quiet tunnel. In: 48th AIAA Aerospace Sciences Meeting Including the New Horizons Forum and Aerospace Exposition. https://doi.org/10.2514/6.2010-1061

13. Chen $X$, Zhu Y, Lee C (2017) Interactions between second mode and low-frequency waves in a hypersonic boundary layer. J Fluid Mech 820:693-735. https://doi.org/10.1017/jfm.2017.233

14. Malik MR, Anderson EC (1991) Real gas effects on hypersonic boundary-layer stability. Phys Fluids 3(5):803-821. https://doi.org/10.1063/1.858012

15. Johnson HB, Seipp TG, Candler GV (1998) Numerical study of hypersonic reacting boundary layer transition on cones. Phys Fluids 10(10):2676-2685. https://doi.org/10.1063/1.869781

16. Bitter NP, Shepherd JE (2015) Stability of highly cooled hypervelocity boundary layers. J Fluid Mech 778:586-620. https://doi.org/10.1017/jfm.2015.358

17. Miró Miró F, Beyak ES, Pinna F, Reed HL (2019) High-enthalpy models for boundary-layer stability and transition. Phys Fluids 31(4):044101. https://doi.org/10.1063/1.5084235

18. Marxen O, laccarino G, Magin TE (2014) Direct numerical simulations of hypersonic boundary-layer transition with finite-rate chemistry. J Fluid Mech 755:35-49. https://doi.org/10.1017/jfm.2014.344

19. Chen X, Wang L, Fu S (2021) Secondary instability of the hypersonic high-enthalpy boundary layers with thermal-chemical nonequilibrium effects. Phys Fluids 33(3):034132. https://doi.org/10.1063/5.0045184

20. Park C (1987) Assessment of two-temperature kinetic model for ionizing air. J Thermophys Heat Transf 3(3):233-244. https://doi.org/10.2514/3.28771

21. Chen X, Wang L, Fu S (2021) Parabolized stability analysis of hypersonic thermal-chemical nonequilibrium boundary-layer flows. AIAA J:1-14. https://doi.org/10.2514/1.J059994

22. Chen X, Fu S (2020) Convergence acceleration for high-order shock-fitting methods in hypersonic flow applications with efficient implicit time-stepping schemes. Comput Fluids 210:104668. https://doi.org/10.1016/j.compfluid.2020. 104668

23. Chang C-L, Malik MR (1994) Oblique-mode breakdown and secondary instability in supersonic boundary layers. J Fluid Mech 273:323-360. https://doi.org/10.1017/S0022112094001965

24. Knisely CP, Zhong X (2017) An investigation of sound radiation by supersonic unstable modes in hypersonic boundary layers. In: 47th AIAA Fluid Dynamics Conference. https://doi.org/10.2514/6.2017-4516

25. Mortensen CH (2018) Toward an understanding of supersonic modes in boundary-layer transition for hypersonic flow over blunt cones. J Fluid Mech 846:789-814. https://doi.org/10.1017/jfm.2018.246

26. Malik MR (2003) Hypersonic flight transition data analysis using parabolized stability equations with chemistry effects. J Spacecr Rocket 40(3):332-344. https://doi.org/10.2514/2.3968

27. Gronvall JE, Johnson HB, Candler GV (2014) Boundary-layer stability analysis of the high enthalpy shock tunnel transition experiments. J Spacecr Rocket 51(2):455-467. https://doi.org/10.2514/1.A32577

28. Zhang C (2017) Research on nonlinear mode interactions relating to supersonic boundary layer transition (in Chinese). Dissertation, Tianjin University. https://kns.cnki.net/kcms/detail/detail.aspx?dbcode=CDFD\&dbname= CDFDLAST2018\&filename=1018011529.nh\& $\mathrm{v}=$ BbSE9BzV5QrWazbLVMbbzguip2fKnGMQLuKrPzks7YwSPzzGaPRqIRIUpsn4b7cc

29. Kendall JM (1975) Wind tunnel experiments relating to supersonic and hypersonic boundary-layer transition. AIAA J 13(3):290-299. https://doi.org/10.2514/3.49694

30. Chuvakhov PV, Fedorov A (2016) Spontaneous radiation of sound by instability of a highly cooled hypersonic boundary layer. J Fluid Mech 805:188-206. https://doi.org/10.1017/jfm.2016.560

31. Bitter NP (2015) Stability of hypervelocity boundary layers. Dissertation, California Institute of Technology. https:// search.proquest.com/docview/1769835659?accountid=14426

\section{Publisher's Note}

Springer Nature remains neutral with regard to jurisdictional claims in published maps and institutional affiliations. 